\title{
Testing for a cultural influence on reading for meaning in the developing brain: the neural basis of semantic processing in Chinese children
}

\author{
Tai-Li Chou 1,2*, Chih-Wei Chen', Li-Ying Fan', Shiou-Yuan Chen ${ }^{3}$ and James R. Booth ${ }^{4}$ \\ Department of Psychology, National Taiwan University, Taipei, Taiwan \\ 2 Neurobiology and Cognitive Science Center, National Taiwan University, Taipei, Taiwan \\ ${ }^{3}$ Department of Early Childhood Education, Taipei Municipal University of Education, Taipei, Taiwan \\ ${ }^{4}$ Department of Communication Sciences and Disorders, Northwestern University, Evanston, IL, USA
}

\section{Edited by:}

Silvia A. Bunge, University of California

Berkeley, USA

\section{Reviewed by:}

Elise Temple, Dartmouth College, USA

Hidenao Fukuyama,

Kyoto University, Japan

*Correspondence:

Tai-Li Chou, National Taiwan University,

Department of Psychology, No. 1,

Sec. 4, Roosevelt Road, Taipei 106,

Taiwan.

e-mail: t/chou25@ntu.edu.tw
Functional magnetic resonance imaging was used to explore the neural correlates of semantic judgments in a group of 8- to 15-year-old Chinese children. Participants were asked to indicate if pairs of Chinese characters presented visually were related in meaning. The related pairs were arranged in a continuous variable according to association strength. Pairs of characters with weaker semantic association elicited greater activation in the mid ventral region (BA 45) of left inferior frontal gyrus, suggesting increased demands on the process of selecting appropriate semantic features. By contrast, characters with stronger semantic association elicited greater activation in left inferior parietal lobule (BA 39), suggesting stronger integration of highly related features. In addition, there was a developmental increase, similar to previously reported findings in English, in left posterior middle temporal gyrus (BA 21), suggesting that older children have more elaborated semantic representations. There were additional age-related increases in the posterior region of left inferior parietal lobule and in the ventral regions of left inferior frontal gyrus, suggesting that reading acquisition relies more on the mapping from orthography to semantics in Chinese children as compared to previously reported findings in English.

Keywords: functional magnetic resonance imaging, semantic, association strength, developmental, children

\section{INTRODUCTION}

Language is acquired without much effort and is associated with maturational changes in the brain (Friederici, 2006). The nature of developmental changes in human cerebral functional organization for language is a core issue in cognitive neuroscience (Brown et al., 2005). This paper focuses on the developmental changes in the neural substrate for semantic processing in reading, which may be somewhat different than spoken language. Emerging evidence of the neural correlates of semantic development mainly comes from English and other alphabetic languages. This paper uses a semantic association task to explore the age effects on the neural correlates of semantic processing in a logographic language, Chinese. The semantic association task has been used to understand the functional architecture of word recognition, identifying brain regions for semantic processing in English in left inferior frontal gyrus (BA 45, 47), left posterior middle temporal gyrus (BA 21), and left inferior parietal lobule (BA 39, 40) in adults (Fletcher et al., 2000; Booth et al., 2002) and in children (Chou et al., 2006a,b).

The role of inferior frontal gyrus in semantic processing has been explored in English by comparing activation to semantic association judgments involving strongly related pairs (e.g., king-queen) versus weakly related pairs (e.g., net-ship). Weaker association produced greater activation in left inferior frontal gyrus as compared to stronger association. Greater activation in left inferior frontal gyrus was suggested to result from the difficulty of selecting appropriate semantic features, as weakly related pairs share few semantic features (Fletcher et al., 2000). In support of this, many studies show greater inferior frontal gyrus activation in more difficult semantic tasks and in tasks with increased retrieval or selection demands. These studies include high vs. low requirement for selection among alternatives (Thompson-Schill et al., 1997, 1999), weak vs. strong association strength (Wagner et al., 2001; Chou et al., 2006a,b), naming low vs. high familiarity objects (Whatmough et al., 2002), generating novel vs. repeated base nouns (Seger et al., 2000), naming low vs. high agreement pictures (Kan and Thompson-Schill, 2004), deep vs. shallow processing of words (Fujii et al., 2002) and producing words for pre-specified semantic categories vs. over-learned letter sequences (Gurd et al., 2002).

A review article recently proposed different cognitive functions for sub-regions of the inferior frontal gyrus (Badre and Wagner, 2007). The anterior ventral region of left inferior frontal gyrus (BA47) may support controlled retrieval of stored semantic representations, whereas the mid-ventral region of left inferior frontal gyrus (BA 45) may support increased selection demands to process active representations. Previous studies using association strength have found greater activation for weaker association pairs in both the anterior ventral region and the mid-ventral region of left inferior frontal gyrus (BA 45, 47) (Badre et al., 2005; Chou et al., 2006a,b). For weaker association pairs, participants may need controlled access to stored conceptual representations to seek for existing associations in verbal semantic memory. Moreover, participants may require a selection process 
that operates post-retrieval to resolve competition among active representations during association judgments.

Studies in English have also shown that weaker association strength between words results in greater activation in left middle temporal gyrus (Chou et al., 2006a; Wible et al., 2006). This region has been implicated in the representation of verbal semantic information (Booth et al., 2002; Blumenfeld et al., 2006). Greater activation in this region for weaker association pairs may result from a more extensive access to semantic representations in order to identify distant relationships (Booth et al., 2007). In contrast, studies in English have shown that word pairs with stronger association result in greater activation in left inferior parietal lobule (Chou et al., 2006a,b; Raposo et al., 2006). Some studies have interpreted the left inferior parietal lobule as involved in feature integration and semantic categorization to form a coherent concept so that relationships between words can be determined (Smith, 1995; Grossman et al., 2003). Left inferior parietal cortex has also been suggested to support the integration of lexical input into the larger units during semantic processing (Lau et al., 2008). Stronger association pairs may involve greater integration because there are more overlapping features between the words or because the shared features are more characteristic of each of the words (Chou et al., 2006a,b). Thus, if there are several overlapping features between words, then semantic integration processes are effectively engaged in the inferior parietal lobule and this results in greater activation in this region. However, if there are few or no overlapping features, then the inferior parietal lobule will not be engaged because it is less or not possible to integrate these features.

Chinese is different from English in the nature of the mapping between orthography and semantics as well as the mapping between orthography and phonology. English has an arbitrary relationship between orthography and semantics at a mono-morphemic level, whereas many Chinese characters are derived from pictures representing meanings or encode meanings by including a semantic radical. There are approximately 200 semantic radicals in Chinese and these units of characters give a clue to the meaning of the character (e.g., category). Thus, Chinese has a more direct mapping between orthography and semantics than English. In support of this, evidence from event-related potential measures suggests earlier involvement of semantics in Chinese compared to English (Zhang et al., 2006). Moreover, spoken Chinese is highly homophonic, with a single syllable shared by many characters. When learning to read, a Chinese child is confronted with the fact that a large number of written characters correspond to the same syllable, and phonological information is insufficient to access semantics of a printed character. There are phonetic radicals, but most of them (about 60\%) provide inconsistent information regarding pronunciation. It is important to explore whether the unique linguistic features of Chinese influence developmental changes in the neural substrate for semantic processing. Based on the more systematic relationship between orthography and semantics and less systematic relationship between orthography and phonology as compared to English, one may expect a larger role for semantics in processing Chinese characters.

Previous English studies examining developmental differences at word level during semantic processing tasks have shown age-related increases in the dorsal region of left inferior frontal gyrus (Schapiro et al., 2004; Brown et al., 2005; Schmithorst et al., 2006; Szaflarski et al., 2006), the anterior region of left inferior parietal lobule (Chou et al., 2006a), and the posterior region of left middle temporal gyrys (Chou et al., 2006a,b; Szaflarski et al., 2006). Limited neuroimaging studies have examined developmental differences in the neural correlates of Chinese language processing (Cao et al., 2009, in press), showing increasing reliance on brain areas involved in visuoorthographic processing and concomitant decreases in reliance on phonology in spelling and rhyming tasks in the visual modality. Reading for meaning places greater demands on the mapping from orthography to semantics at word level in Chinese (Leck et al., 1995; Zhou and Marslen-Wilson, 2000; Feng et al., 2001), and therefore one may expect that the unique structure of Chinese influences developmental trajectories. Reading development of Chinese may be characterized by decreased reliance on phonology due to many homophones in Chinese, making it more efficient to access meaning through the connection between orthography and semantics rather than the connection of orthography to phonology to semantics (Peng et al., 1985; Song et al., 1995; Meng et al., 2007).

The current study examined the neural substrate of developmental changes during semantic processing in Chinese children (8-to 15-year olds). We manipulated semantic relatedness by varying the 'free association strength' between pairs of visually presented Chinese characters (Hue et al., 2005). From aforementioned studies, we expected weaker association to produce greater activation in the ventral region of left inferior frontal gyrus, and stronger association to produce greater activation in left inferior parietal lobule. Moreover, we investigated whether there were similarities and differences on reading for meaning in the developing brain in Chinese as compared to those reported in English, with an attempt to differentiate between brain processes inherent across languages from those specific to the unique structure of a language.

\section{MATERIALS AND METHODS PARTICIPANTS}

Thirty-three native speakers of Chinese (mean age $=12.3$, standard deviation $=1.8,16$ females) in Taiwan participated in the functional magnetic resonance imaging (fMRI) study. The participants were all monolingual. The distribution of ages and genders is shown in Table 1. Parents were given an informal interview to insure that their children met the following inclusionary criteria: (1) right-handedness, (2) normal hearing and normal or corrected-to-normal vision after an examination of vision by experimenters, (3) free of neurological disease or psychiatric disorders, (4) not taking medication affecting the central nervous system, (5) no history of intelligence, reading, or oral-language deficits, and (6) no learning disability or attention deficit hyperactivity disorder. After the administration of the interview, informed consent was obtained. Informed consent procedures were approved by the Institutional Review Board at the National Taiwan University Hospital. Standardized intelligence testing was then administered, using the Wechsler Intelligence Scale for Children

Table 1 |The distribution of ages and genders.

\begin{tabular}{lllll}
\hline & Ages 8-9 & Ages 10-11 & Ages 12-13 & Ages 14-15 \\
\hline Female & 2 & 6 & 4 & 4 \\
Male & 2 & 4 & 7 & 4
\end{tabular}


(WISC-III) Chinese version (The Psychological Corporation, 1999). Participants's standard scores (mean \pm SD) were $113 \pm 10$ on the verbal scale and $114 \pm 11$ on the performance scale.

\section{FUNCTIONAL ACTIVATION TASKS}

The children were given two practice sessions, one outside the scanner and the other in the scanner, to make sure that they understood the task. The practice items were different stimuli than those used in fMRI sessions. Each participant was at least $80 \%$ correct for each condition separately for both practice sessions. In the meaning judgment task, two visual Chinese characters were presented sequentially and the participant had to determine whether the character pair was related in meaning. Trials lasted $4500 \mathrm{~ms}$ and consisted of a solid square $(500 \mathrm{~ms})$, followed by the first character $(800 \mathrm{~ms})$, a $200-\mathrm{ms}$ blank interval, and the second character for $3000 \mathrm{~ms}$. The duration of $800 \mathrm{~ms}$ for the first word and a 200-ms ISI was the same as our previous English study (Chou et al., 2006a). The duration of $3 \mathrm{~s}$ for the second word was based on our previous Chinese study testing adults, healthy children, and children with smaller vocabulary sizes (Lee et al., in press). The upper bound of the mean $+2.5 \mathrm{SD}$ of reaction time for children with smaller vocabulary sizes was close to $3 \mathrm{~s}$. Thus, the duration of $3 \mathrm{~s}$ for the second word was chosen for a previous adult study (Chou et al., in press) and for this child study. The participant was instructed to make a response during the presentation of the second character.

Forty-eight character pairs were semantically related according to their free association values (mean $=0.14, \mathrm{SD}=0.13$, ranging from 0.73 to 0.01 ) (Hue et al., 2005). The Chinese norms (Hue et al., 2005) were created by presenting 100 native Mandarin-Chinese speakers with a list of target words and asking them to generate the first word that came to mind. For example, if 40 out of the 100 participants generated the same word to a given target, the association strength was 0.4 for the word pair. This same procedure has been used in English (Nelson et al., 1998). Even though some of the word pairs had low association values in the related pairs, on average, the participants in our study were able to determine they were related. For example, the average accuracy for the word pair with association strength 0.01 was above $70 \%$. Character pairs were arranged in a continuous variable according to association values. The distribution of association values was positively skewed so a logarithmic transformation was used (Howell, 2006). When values are near-zero, one can use $\log (X+1)$ to perform a logarithmic transformation, but we used $\log (100 X+1)$ in order to get a positive number after transformation (mean $=1.04, \mathrm{SD}=0.36$, ranging from 1.86 to 0.30 ). Such a logarithmic transformation has been shown to be suitable for item-level parametric analyses in developmental studies (Bolger et al., 2008). In addition, 24 word pairs were semantically unrelated with zero association values. The participants were instructed to quickly and accurately press with their right hand the yes button to the related pairs and the no button to the unrelated pairs.

The perceptual control condition had 24 pairs of non-characters. Non-characters were created by replacing radicals of real characters with other radicals that did not form real Chinese characters. Noncharacters were larger ( 50 font size) than real characters ( 40 font size) in order to encourage participants to perform the task based on the recognition of low level visual similarity and not on the extraction of semantic information. The size was a cue for participants to judge the stimuli perceptually. For the perceptual control condition, trials consisted of a solid square $(500 \mathrm{~ms})$, followed by the first noncharacter $(800 \mathrm{~ms})$, a $200-\mathrm{ms}$ blank interval, and the second noncharacter for $3000 \mathrm{~ms}$. Participants determined whether the pair of stimuli were identical or not by pressing a yes or no button with their right hand. There were also 24 baseline events as 'null' trials so that we could better deconvolve the response to the lexical and perceptual trials. The participant was instructed to press a button when a solid square (1300 ms) at the center of the visual field turned to a hollow square $(3000 \mathrm{~ms})$ after a blank interval $(200 \mathrm{~ms})$. In order to control for visual-orthographic information between real characters and non-characters, we compared the related or unrelated conditions to the perceptual control condition for the fMRI analyses.

\section{STIMULUS CHARACTERISTICS}

Several lexical variables were controlled across the related and unrelated conditions. First, all Chinese characters were monosyllabic. Second, the first character and the second character did not share radicals. Third, the first character and the second character did not form a word (Wu and Liu, 1987; Sinica Corpus, 1998). Fourth, the number of nouns, verbs, adjectives, adverbs was similar for strong $(48 \%, 23 \%, 29 \%$, and $0 \%)$, weak $(48 \%, 23 \%, 25 \%$, and $4 \%)$ and unrelated pairs $(50 \%, 23 \%, 21 \%$, and $6 \%)$, based on their most frequent usage in Academia Sinica balanced corpus (Sinica Corpus, 1998). In addition, we split the related condition into strong and weak pairs, each pair with 24 trials, and calculated two word (first, second) by three relatedness (strong, weak, unrelated) ANOVAs on stimulus characteristics. First, characters were matched for visual complexity (in terms of strokes per character) across conditions. The main effect of relatedness, $F(2,138)=0.68, p>0.05$, nor its interaction with word was significant, $F(2,138)=2.85, p>0.05$. In addition, the correlation of visual complexity (first or second words) with association strength was not significant, $r=-0.20, p>0.05$. Second, characters were matched for written frequency for adults (Wu and Liu, 1987) and written familiarity for children across conditions. For written frequency, the main effect of relatedness, $F(2,138)=1.00, p>0.05$, nor its interaction with word was significant, $F(2,138)=2.41, p>0.05$. In addition, the correlation of frequency (first or second words) with association strength was not significant, $r=-0.02, p>0.05$. Familiarity scores were obtained from pre-tests in which all the characters were rated on a 7 -point scale by 30 age-matched children who were native Mandarin speakers from Taiwan. The instruction for written familiarity asked the children how often they saw the word in books, newspaper, and magazines. The 30 children were equally distributed across the 8 - to 15 -year-old age range. For written familiarity, the main effect of relatedness, $F(2,138)=0.7, p>0.05$, nor its interaction with word was significant, $F(2,138)=2.62, p>0.05$. Finally, the semantic relation for related pairs (Lee et al., in press) was a dichotomous scale (categorical coding:1, functional coding:0). The point-biserial correlation of the measure of semantic relation with association strength was not significant, $r=-0.05, p>0.05$, indicating that association effects should not be due to semantic relation differences.

\section{MRI DATA ACOUISITION}

Participants lay in the scanner with their head position secured. An optical response box was placed in the participants' right hand. The 
head coil was positioned over the participants' head. Participants viewed visual stimuli projected onto a screen via a mirror attached to the inside of the head coil. This study adopted an event-related design. Each participant performed two functional runs. Each run took $4.7 \mathrm{~min}$.

All images were acquired using a 3 Tesla Siemens scanner. Gradient-echo localizer images were acquired to determine the placement of the functional slices. For the functional imaging studies, a susceptibility weighted single-shot EPI (echo planar imaging) method with BOLD (blood oxygenation level-dependent) was used. Functional images were interleaved from bottom to top collected parallel to the AC-PC plane. The following scan parameters were used: $\mathrm{TE}=24 \mathrm{~ms}$, flip angle $=90^{\circ}$, matrix size $=64 \times 64$, field of view $=25.6 \mathrm{~cm}$, slice thickness $=3 \mathrm{~mm}$, number of slices $=34$; $\mathrm{TR}=2000 \mathrm{~ms}$. Each participant performed two 4.7-min functional runs. Each functional run had 4 dummy volumes discarded from fMRI analysis and 136 image volumes used for fMRI analysis. In addition, a high resolution, T1 weighted 3D image was acquired $\left(\mathrm{TR}=1560 \mathrm{~ms}, \mathrm{TE}=3.68 \mathrm{~ms}\right.$, flip angle $=15^{\circ}$, matrix size $=256 \times 256$, field of view $=25.6 \mathrm{~cm}$, slice thickness $=1 \mathrm{~mm}$, number of slices $=192$ ). The orientation of the 3D image was identical to the functional slices. The task was administered in a pseudorandom order for all subjects, in which the order of related, unrelated, perceptual, and baseline trials was optimized for eventrelated design (Burock et al., 1998). We used the Optseq script for randomized event-related design (http://surfer.nmr.mgh.harvard. edu/optseq, written by D. Greve, Charlestown, MA, USA) that implemented Burock et al. (1998)'s approach.

\section{IMAGE ANALYSIS}

Data analysis was performed using SPM2 (Statistical Parametric Mapping). The functional images were corrected for differences in slice-acquisition time to the middle volume and were realigned to the first volume in the scanning session using affine transformations. No participant had more than $3 \mathrm{~mm}$ of movement in any plane. We judged a 3-mm criterion to be appropriate because this was less than the in plane resolution of acquisition $(4 \mathrm{~mm})$. The average estimated motion over the whole run was small in all directions $(x=0.02 \mathrm{~mm} ; y=0.07 \mathrm{~mm} ; z=0.04 \mathrm{~mm}$ ). In addition, the maximum displacement in any direction was small for each trial type (related $=0.019 \mathrm{~mm}$, perceptual $=0.021 \mathrm{~mm}$, baseline $=0.021 \mathrm{~mm}$, unrelated $=0.021 \mathrm{~mm}$ ). There were no significant differences in maximum movement across trial types and there was not correlation of $\mathrm{x}, \mathrm{y}$ or $\mathrm{z}$ movement with age. Co-registered images were normalized to the MNI (Montreal Neurological Institute) average template (12 linear affine parameters for brain size and position, 8 non-linear iterations and $2 \times 2 \times 2$ nonlinear basis functions). We did not use a child template because given the age of our participants and the voxel size it was appropriate to use an adult template (Burgund et al., 2002). This also allowed for comparison of the results of the present study with other published adult studies. Statistical analyses were calculated on the smoothed data (10 $\mathrm{mm}$ isotropic Gaussian kernel), with a high pass filter (128 s cutoff period).

Data from each participant was entered into a general linear model using an event-related analysis procedure (Penny and Holmes, 2003). Character pairs were treated as individual events for analysis and modeled using a canonical HRF (Hemodynamic
Response Function). There were four event types: related, unrelated, perceptual, and baseline. For the related pairs, association strength was an item-level parametric modulator in order to differentiate semantic relatedness as a continuous variable according to log transformed free association strength (Hue et al., 2005). The analysis of association strength was based on the related pairs and was not in comparison to baseline (also see Bolger et al., 2008). The resulting model coefficients for individual subjects were entered into subsequent second-order random-effects analyses in a whole brain analysis. All reported areas of activation were significant using $p<0.05$ corrected for FDR (false discovery rate) at the voxel level with a cluster size greater than or equal to 10 voxels.

Random-effects analysis using one-sample $t$-tests across all participants was used to determine whether activation during a contrast was significant (i.e., parameter estimates were reliably greater than zero). First, we compared the related and unrelated pairs separately to the perceptual control condition, and the related to the unrelated pairs in a whole brain analysis. Second, we examined the effects of association strength for the related pairs, including reaction times as an item-based (within-subject) covariate. This allowed us to examine the association effects that were independent of reaction time differences. Positive effects indicated greater activation for related pairs with stronger association strength, whereas negative effects indicated greater activation for related pairs with weaker association strength. For the effects of association strength, we used the adult study (Chou et al., in press) to determine if a similar effect of association strength was present in children. An inclusive mask ( $p<0.005$ uncorrected) of left inferior parietal activation from the adult study was used to test for stronger association in this child study. An inclusive mask $(p<0.005$ uncorrected) of left inferior frontal activation from the adult study was used to test for weaker association in this child study. Third, we used multiple regression to correlate the continuous variable of age in months with signal intensity for the related minus unrelated contrast, including accuracy or reaction time as a betweensubject covariate. This allowed us to examine age-related increases or decreases in activation that were independent of accuracy or reaction time differences. We extracted the beta values from the peak voxels of brain regions to visualize correlations for these age analyses.

\section{RESULTS}

\section{BEHAVIORAL PERFORMANCE}

Because $6 \%$ of accuracy values were located outside 2.5 SD from the group mean for each condition, trimming these outliers was applied to accuracy analysis. The subject-based accuracy (mean \pm SD) for the related and unrelated conditions was $88 \pm 10 \%$ and $96 \pm 6 \%$, respectively, with the related condition being less accurate than the unrelated condition, a paired $t(32)=4.31, p<0.01$. The subject-based reaction times (mean $\pm \mathrm{SD}$ ) measured from the onset of the second stimulus for the related and unrelated conditions were $1012 \pm 244 \mathrm{~ms}$ and $992 \pm 221 \mathrm{~ms}$, respectively, with no significant difference, a paired $t(32)=1.11, p=0.28$. The correlations of age with accuracy and reaction times were not significant for the related pairs $[r(33)=0.216, p=0.228$; and $r(33)=-0.338$, $p=0.054$, respectively]. Because character pairs were arranged in a continuous variable according to association strength, we calculated correlations between association strength for related pairs and behavioral performance. The item-based correlation between 
accuracy and association strength was not significant, $r(48)=0.27$, $p=0.06$; and the item-based correlation between reaction times and association strength was negative, $r(48)=-0.39, p<0.01$. The subject-based accuracy and reaction times (mean \pm SD) for the perceptual control were $99 \pm 1 \%$ and $687 \pm 152 \mathrm{~ms}$, respectively. The subject-based accuracy and reaction times (mean \pm SD) for the baseline were $99 \pm 2 \%$ and $615 \pm 155 \mathrm{~ms}$, respectively.

\section{BRAIN ACTIVATION PATTERNS}

The presentation of the results will focus on brain regions that have been implicated in previous studies of semantic processing, namely left inferior frontal gyrus, posterior middle temporal gyrus, and inferior parietal lobule. All activation differences are reported in the tables. Because no significant differences were found between the analysis of correct responses alone and the analysis that includes all responses, only results from the analysis with all responses are presented to equate the statistical power between conditions with different accuracies (Bitan et al., 2007). This analysis was done by separately modeling correct and incorrect responses, and only examining condition differences for correct responses. We examined condition differences using correct responses for related versus perceptual, unrelated versus perceptual and related versus unrelated. The peak coordinates and corresponding $Z$ values were similar for all responses and for correct responses only.

Table 2 shows greater activation for the related or unrelated pairs compared to the perceptual control condition, and for the related compared to the unrelated pairs. Both related and unrelated pairs produced greater activation in left inferior frontal gyrus (IFG, BA 47,45 ) and left posterior middle temporal gyrus (MTG, BA 21) as compared to the perceptual control condition. The direct comparison of the related and unrelated pairs produced greater activation in left inferior frontal gyrus (BA 47, 45), left posterior middle temporal gyrus (BA 21), and left inferior parietal lobule (IPL, BA 39).

The effects of semantic association strength for the related pairs, partialing out the effect of reaction times in the scanner as a within-subject covariate, are shown in Table 3. Stronger association

Table 2 | Greater activation for the related or unrelated conditions compared to the perceptual control condition. The direct comparison between the related and unrelated conditions is also presented.

\begin{tabular}{|c|c|c|c|c|c|c|c|c|}
\hline Condition & Regions & $\mathbf{H}$ & BA & $z$-test & Voxels & $x$ & $y$ & $z$ \\
\hline \multirow[t]{4}{*}{ Related-Perceptual } & Inferior frontal gyrus & $L$ & 47 & 7.81 & 1677 & -42 & 26 & 0 \\
\hline & Inferior frontal gyrus & $\mathrm{L}$ & 45 & & & -48 & 21 & 12 \\
\hline & Medial frontal gyrus & $L$ & 6 & 6.66 & 1308 & -6 & 24 & 42 \\
\hline & Caudate & $\mathrm{L}$ & - & 5.73 & 1602 & -12 & 6 & 9 \\
\hline \multirow[t]{5}{*}{ Unrelated-Perceptual } & Inferior frontal gyrus & $\mathrm{L}$ & 47 & 5.78 & 1000 & -42 & 24 & 0 \\
\hline & Inferior frontal gyrus & $\mathrm{L}$ & 45 & & & -48 & 24 & 10 \\
\hline & Middle frontal gyrus & $L$ & 46 & & & -48 & 21 & 27 \\
\hline & Medial frontal gyrus & $\mathrm{L}$ & 6 & 4.91 & 253 & -3 & 9 & 63 \\
\hline & Middle temporal gyrus & $L$ & 21 & 3.44 & 19 & -57 & -48 & 3 \\
\hline & Medial frontal gyrus & $L$ & 6 & 4.38 & 358 & -9 & 6 & 72 \\
\hline & Middle temporal gyrus & $L$ & 21 & 3.75 & 56 & -60 & -42 & -3 \\
\hline & Middle frontal gyrus & $L$ & 6 & 3.74 & 49 & -42 & -3 & 54 \\
\hline & Inferior parietal lobule & $L$ & 39 & 3.47 & 27 & -42 & -66 & 36 \\
\hline
\end{tabular}

Note. H: hemisphere, L: left, R: right, BA: Brodmann's Area. Coordinates of activation peak(s) within a region based on a z-test are given in the MNI stereotactic space $(x, y, z)$. Voxels: number of voxels in cluster at $p<0.05$ FDR (false discovery rate) corrected, only clusters greater than or equal to 10 are presented.

Table 3 | The effects of semantic association for related pairs.

\begin{tabular}{|c|c|c|c|c|c|c|c|c|}
\hline Modulation & Regions & $\mathbf{H}$ & BA & z-test & Voxels & $x$ & $y$ & $\mathbf{z}$ \\
\hline \multirow[t]{2}{*}{ Stronger Association } & Inferior parietal lobule & $\mathrm{L}$ & 39 & 4.22 & 65 & -42 & -64 & 33 \\
\hline & Inferior parietal lobule & $\mathrm{L}$ & 40 & & & -44 & -59 & 30 \\
\hline Weaker Association & Inferior frontal gyrus & $\mathrm{L}$ & 45 & 3.45 & 14 & -48 & 22 & 18 \\
\hline
\end{tabular}

Note. See Table 2 note. The activation maps of stronger and weaker association (Chou et al., in press) were used as inclusive masks ( $<<0.005$ uncorrected) for the stronger and weaker association analyses in this study, respectively. 
produced greater activation in left inferior parietal lobule (BA 39, 40) (see Figure 1A). Weaker association produced greater activation in left mid ventral inferior frontal gyrus (BA 45) (see Figure 1B).

The correlations between age in months and activation, partialing out the effect of accuracy or reaction time in the scanner as a between-subject covariate, are shown in Table 4. Both correlations show that increasing age was correlated with greater activation for the related pairs compared to the unrelated pairs in the anterior and mid ventral regions of left inferior frontal gyrus (BA 45, 47), left posterior middle temporal gyrus (BA 21), and left inferior parietal lobule (BA 39) (Figure 2). For descriptive purposes, we visualize the age effects by the correlations between age in months and beta values from the peak voxels of these three regions for the related pairs (correlations in IFG, MTG, and IPL were $r=0.44 ; r=0.41 ; r=0.43$, respectively), as well as for the unrelated pairs (correlations in IFG, MTG, and IPL were $r=0.31 ; r=0.05 ; r=0.07$, respectively). There were no negative age effects for the related minus unrelated pairs.

\section{DISCUSSION}

The neural correlates of semantic processing in children (8- to 15 -year olds) to visually presented Chinese characters were examined

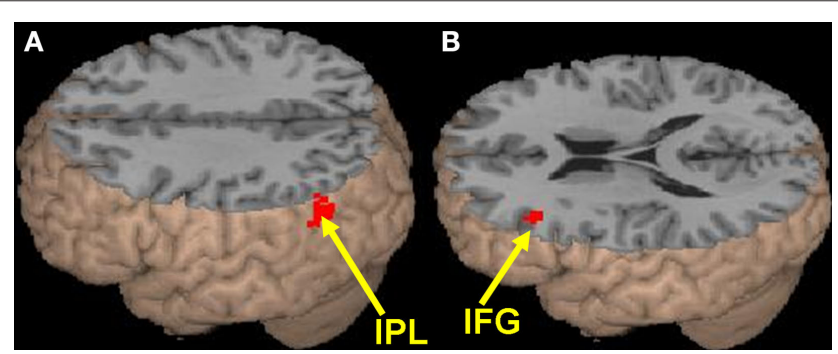

FIGURE 1 |The effects of semantic association strength. (A) Stronger association produced greater activation for the related pairs in left inferior parietal lobule (IPL, BA 39, 40). (B) Weaker association produced greater activation for the related pairs in the mid ventral portion of left inferior frontal gyrus (IFG, BA 45). with a task requiring association judgments as to whether character pairs were related in meaning. In order to more effectively measure activation within the semantic system, we manipulated the strength of association between the words in related pairs. Similar to previous child studies on English (Chou et al., 2006a,b), in Chinese stronger semantic association produced greater activation in left inferior parietal lobule (BA 39, 40), whereas weaker semantic association produced greater activation in the mid ventral region of left inferior frontal gyrus (BA 45). Regarding age effects on semantic processing, developmental increases in left posterior middle temporal gyrus were similar to those previously reported for English (BA 21) (Chou et al., 2006a,b; Szaflarski et al., 2006). However, developmental increases in left inferior parietal activation were more posterior in Chinese (BA 39) than in previous studies on English (BA 40) (Chou et al., 2006a), and left inferior frontal activation were more ventral in Chinese (BA 45, 47) than in previous studies on English (BA 6, 9) (Schapiro et al., 2004; Brown et al., 2005; Schmithorst et al., 2006; Szaflarski et al., 2006). After a discussion of the association strength effects, we will turn to a consideration of the developmental differences.

Stronger semantic association produced greater activation in left inferior parietal lobule (BA 39, 40). Activation in this region has previously been identified in semantic association tasks in English children and Chinese adults (Chou et al., 2006a,b, in press). Greater activation in left inferior parietal lobule has been interpreted as evidence of semantic integration (Thompson et al., 2007). Left inferior parietal cortex has also been suggested to support the integration of lexical input into the larger units during semantic processing (Lau et al., 2008). Stronger association pairs may allow for greater integration because there are more overlapping features between the words or because the shared features are more characteristic of each of the words (Chou et al., 2006a,b). Greater integration for stronger association pairs may account for the increase in left inferior parietal lobule activation with increasing association strength in Chinese. Thus, the stronger association effect was similar between Chinese and English as well as between adults and children.

Table 4 | Increasing activation with age for the related pairs compared to the unrelated pairs, partialing out the effect of accuracy (ACC) or reaction time (RT).

\begin{tabular}{|c|c|c|c|c|c|c|c|c|}
\hline Increase with age & Regions & $\mathbf{H}$ & BA & $z$-test & Voxels & $x$ & $y$ & $z$ \\
\hline \multirow[t]{4}{*}{ Partial for ACC } & Caudate & L & - & 4.51 & 546 & -9 & 6 & 9 \\
\hline & Inferior frontal gyrus & L & 45 & 4.31 & 259 & -51 & 27 & 10 \\
\hline & Inferior parietal lobule & L & 39 & 4.02 & 52 & -40 & -69 & 34 \\
\hline & Middle temporal gyrus & L & 21 & 3.44 & 19 & -58 & -39 & -3 \\
\hline & Caudate & L & - & 4.47 & 313 & -9 & 9 & 9 \\
\hline & Medial frontal gyrus & L & 6 & 4.26 & 206 & -9 & 9 & 72 \\
\hline & Middle frontal gyrus & L & 6 & 3.62 & 25 & -42 & -3 & 54 \\
\hline & Middle temporal gyrus & L & 21 & 3.57 & 32 & -58 & -42 & -1 \\
\hline & Inferior parietal lobule & $\mathrm{L}$ & 39 & 3.54 & 19 & -36 & -69 & 36 \\
\hline
\end{tabular}

Note. See Table 2 note. 


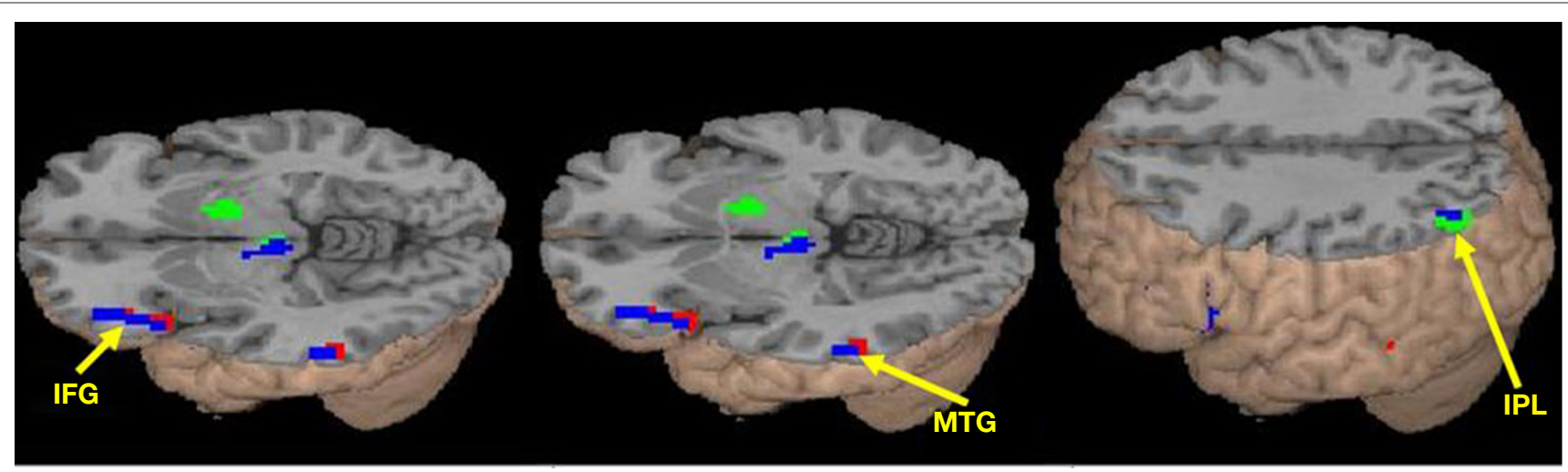

IFG

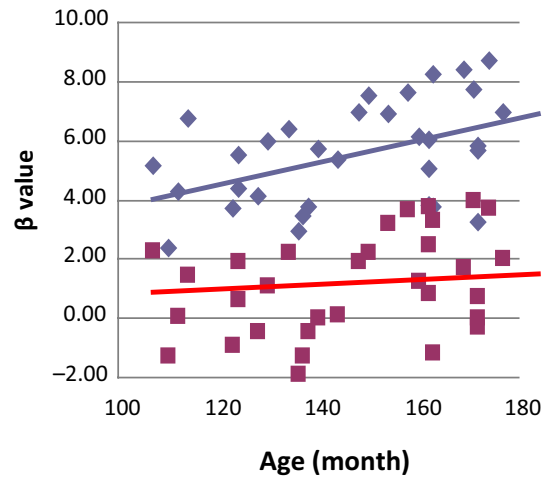

MTG

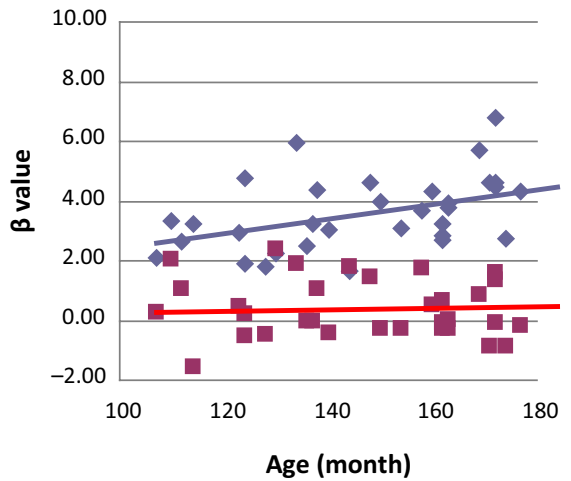

IPL

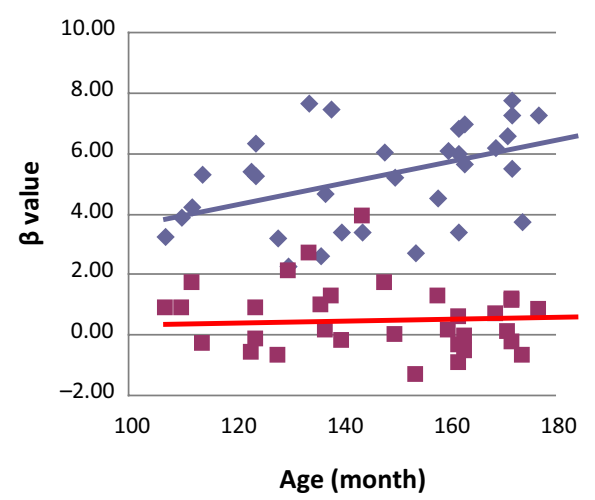

FIGURE 2 |The effects of age, partialed for accuracy (green) or reaction time (red), for the related minus unrelated pairs. The overlap between the two maps is represented in blue, showing that increasing age was correlated with greater activation in the anterior and mid ventral regions of left inferior frontal gyrus (IFG), left posterior middle temporal gyrus (MTG), and the posterior region of left inferior parietal lobule (IPL).
For descriptive purposes, the graphs below are used to visualize the whole brain data that are shown above. The scatterplots of the correlation of age in months with the related (blue) and unrelated (red) conditions in these three regions are presented below the brain images. Beta values were taken from the peak voxel of the related minus unrelated contrast partialed for reaction time.
We also found that weaker semantic association produced greater activation in the mid-ventral region of left inferior frontal gyrus (BA 45), similar to those reported in English children (Chou et al., 2006a) and Chinese adults (Chou et al., in press). Previous studies in English suggest that this region is involved in effortful semantic processing, particularly when there is increased demands on the process of selecting relevant semantic knowledge or when comparing words along semantic features (Thompson-Schill et al., 1997, 1999; Fletcher et al., 1998; Whatmough et al., 2002; Blumenfeld et al., 2006). Badre and Wagner (2007) propose that the anterior ventral region of left inferior frontal gyrus (BA 47) may support controlled access to stored semantic representations, whereas the mid-ventral region of left inferior frontal gyrus (BA 45) may support increased selection demands to process active representations. Of particular relevance to the current study, semantic judgments to weaker association pairs produced greater activation in the mid-ventral region of left inferior frontal gyrus as compared to stronger association pairs. Greater activation for weaker association pairs could result from increased demands on the selection of appropriate semantic features in Chinese. Thus, the weaker association effect was similar between Chinese and English as well as between adults and children.
Developmental increases in left posterior middle temporal gyrus in Chinese are consistent with English studies showing age-related increases in this region (Chou et al., 2006a,b; Szaflarski et al., 2006). Previous child studies in English and Chinese implicate left posterior middle temporal gyrus in semantic processes (Chou et al., 2006a; Cao et al., 2009). Several studies suggest that the best candidate for the storage of lexical representations is in left posterior middle temporal gyrus (Hickok and Poeppel, 2004, 2007; Martin, 2007). Greater activation over age in this region may be associated with increasing elaboration of semantic representations, i.e. a greater number of semantic representations with more interconnections between these representations. Behavioral research shows that as vocabulary knowledge increases, the child's semantic system is gradually elaborated due to a greater number of conceptual links in Chinese and English (McGregor and Appel, 2002; McGregor et al., 2002; Lee et al., in press). Therefore, the developmental increase in left posterior middle temporal gyrus may reflect a general process that is universal across languages.

Developmental increases in left inferior parietal lobule [-40, -69, 34 in the present Chinese study are more posterior than developmental effects in this region $[-45,-33,40]$ reported in previous 
studies of English using the same visual semantic task (Chou et al., 2006a). The anterior region of the left inferior parietal lobule has been implicated as part of the phonological loop (Paulesu et al., 1993) or the phonological store (Chen and Desmond, 2005), whereas the posterior region of left inferior parietal lobule has been suggested to support the integration of lexical input into larger units for semantic processing (Lau et al., 2008). Because reading for meaning places greater demands on the mapping from orthography to semantics at word level in Chinese (Leck et al., 1995; Zhou and Marslen-Wilson, 2000; Feng et al., 2001), developmental increases in the posterior region of left inferior parietal lobule may be related to more extensive semantic integration in older children. This suggestion is supported by our finding reported above that stronger association pairs, which can be more thoroughly integrated, also produced greater activation than weaker association pairs in the posterior region of left inferior parietal lobule. The developmental increases in the anterior region of left inferior parietal lobule in English may be due to greater involvement of phonological processing when reading in an alphabetic language.

In contrast to developmental studies of English, our study showed an age-related increase in ventral regions of left inferior frontal gyrus for Chinese children. Previous studies in English have shown developmental increases in more dorsal frontal regions in auditory narrative comprehension at $[-42,7,30]$ (Schmithorst et al., 2006) and in verb generation in response to a noun at $[-49$, 3, 39] (Brown et al., 2005), at [-46, 7, 25] (Schapiro et al., 2004), and at $[-47,3,30]$ (Szaflarski et al., 2006). Our coordinates in left inferior frontal gyrus at $[-51,27,10]($ BA 45$)$ and at $[-48,21,-3]$ (BA 47) showing developmental effects in Chinese are substantially ventral to the coordinates reported in English studies. The subparts of left inferior frontal gyrus have been associated with distinct cognitive processes involved in language tasks. In general, the dorsal region of left inferior frontal gyrus is thought to be specialized for processing phonological representations, while the ventral region of left inferior frontal gyrus is proposed to be specialized for manipulating semantic representations (Poldrack et al., 1999), including controlled retrieval and selection mechanisms in ventral prefrontal regions (Badre, et al., 2005). Because reading for meaning places greater demands on mapping from orthography to semantics in Chinese (Leck et al., 1995; Zhou and Marslen-Wilson, 2000; Feng et al., 2001), older children may have learned to engage more thoroughly in controlled retrieval and selection of semantic knowledge at word level. The developmental increases in dorsal regions of left inferior frontal gyrus in English may reflect greater phonological involvement in alphabetic languages in older children.

\section{REFERENCES}

Badre, D., Poldrack, R. A., Pare-Blagoev, E. J., Insler, R. Z., and Wagner, A. D. (2005). Dissociable controlled retrieval and generalized selection mechanisms in ventrolateral prefrontal cortex. Neuron 47, 907-918.

Badre, D., and Wagner, A. D. (2007). Left ventrolateral prefrontal cortex and the cognitive control of memory. Neuropsychologia 45, 2883-2901.

Bitan, T., Burman, D. D., Chou, T. L., Lu, D., Cone, N. E., Cao, F., Bigio, J. D.,

There are two potential limitations of the study. First, the related condition had twice as many trials as all other conditions, possibly resulting in higher signal-to-noise ratio in the related condition. We checked that in three critical regions (left inferior frontal gyrus, left middle temporal gyrus, and left inferior parietal lobule) signal was greater for the related than the unrelated condition. However, this difference should not have affected the main results of the paper as the strength of association effect was examined within the related trials, and age effects were examined for the related condition. Second, it is possible that the non-characters for the perceptual control condition could have been in part processed linguistically because radicals of real characters were replaced with other radicals to form non-characters. However, the comparison of the related or unrelated to the perceptual condition revealed activation in both left inferior frontal gyrus and left middle temporal gyrus, suggesting that real characters more robustly activated the semantic system than non-characters. Moreover, our association and age analyses examined only the related characters. Thus, the non-characters would be unlikely to have influenced the results of these analyses.

In conclusion, our study of Chinese children showed association strength effects in left inferior parietal lobule and in mid-ventral regions of left inferior frontal gyrus. These brain regions have been implicated in semantic integration and selection, respectively. We additionally showed that there were agerelated increases in left posterior middle temporal gyrus, similar to those reported in previous studies of English, suggesting that older children have more elaborated semantic representations across scripts. Because Chinese has a more consistent relationship in the mapping between orthography and semantics and a less consistent relationship in mapping between orthography and phonology, the developmental increases in the posterior region of left inferior parietal lobule and in the ventral regions of left inferior frontal gyrus suggests that reading acquisition relies more on the mapping from orthography to semantics in Chinese children at word level.

\section{ACKNOWLEDGMENTS}

This research was supported by a grant from the National Science Council of Taiwan (NSC 96-2413-H-002-023-MY2) to Tai-Li Chou. This research was also supported by grants from the National Institute of Child Health and Human Development (HD042049) to James R. Booth. This research was supported in part by the Department of Medical Imaging and 3T MRI Lab in National Taiwan University Hospital.

consistency in cortex for children with and without reading impairment. Neuropsychologia 46, 3210-3224.

Booth, J. R., Bebko, G., Burman, D. D., and Bitan, T. (2007). Children with reading disorder show modality independent brain abnormalities during semantic tasks. Neuropsychologia 45, 775-783.

Booth, J. R., Burman, D. D., Meyer, J. R., Gitelman, D. R., Parrish, T. B., and Mesulam, M. M. (2002). Modality independence of word comprehension. Hum. Brain Mapp. 16, 251-261.
Brown, T. T., Lugar, H. M., Coalson, R. S., Miezin, F. M., Petersen, S. E., and Schlaggar, B.L. (2005). Developmental changes in human cerebral functional organization for word generation. Cereb. Cortex 15, 275-290.

Burgund, E. D., Kang, H. C., Kelly, J. E., Buckner, R. L., Snyder, A. Z., Petersen, S. E., and Schlaggar, B. L. (2002). The feasibility of a common stereotactic space for children and adults in fMRI studies of development. Neuroimage 17, 184-200. 
Burock, M.A., Buckner, R. L., Woldorff,M. G., Rosen, B. R., and Dale, A. M. (1998). Randomized event-related experimental designs allow for extremely rapid presentation rates using functional MRI. Neuroreport 9, 3735-3739.

Cao, F., Lee, R., Shu, H., Yang, Y., Xu, G., Li, K., and Booth, J. R. (in press). Cultural constraints on brain development: evidence from a developmental study of visual word processing in Mandarin Chinese. Cereb. Cortex.

Cao, F., Peng, D., Liu, L., Jin, Z., Fan, N., Deng, Y., and Booth, J. R. (2009). Developmental differences of neurocognitive networks for phonological and semantic processing in Chinese word reading. Hum. Brain Mapp. 30, 797-809.

Chen, S. H., and Desmond, J. E. (2005). Temporal dynamics of cerebro-cerebellar network recruitment during a cognitive task. Neuropsychologia 43, 1227-1237.

Chou, T.L., Booth, J. R., Bitan, T., Burman, D. D., Bigio, J. D., Cone, N. E., Lu, D., and Cao, F. (2006a). Developmental and skill effects on the neural correlates of semantic processing to visually presented words. Hum. Brain Mapp. 27, 915-924.

Chou, T. L., Booth, J. R., Burman, D. D., Bitan, T., Bigio, J. D., Lu, D., and Cone, N. E. (2006b). Developmental changes in the neural correlates of semantic processing. Neuroimage 29, 1141-1149.

Chou, T. L., Chen, C. W., Wu, M. Y., and Booth, J. R. (in press). The role of inferior frontal gyrus and inferior parietal lobule in semantic processing of Chinese characters. Exp. Brain Res.

Feng G., Miller, K., Shu, H., and Zhang, H. (2001). Rowed to recovery: the use of phonological and orthographic information in reading Chinese and English. J. Exp. Psychol. Learn. Mem. Cogn. 27, 1079-1100.

Fletcher, P. C., Shallice, T., and Dolan, R. J. (1998). The functional roles of prefrontal cortex in episodic memory. I. Encoding. Brain 121, 1239-1248.

Fletcher, P. C., Shallice, T., and Dolan, R. J. (2000). "Sculpting the response space" - an account of left prefrontal activation at encoding. Neuroimage 12, 404-417.

Friederici, A. D. (2006). The neural basis of language development and its impairment. Neuron 52, 941-952.

Fujii, T., Okuda, J., Tsukiura, T., Ohtake, H., Suzuki, M., Kawashima, R., Itoh, M., Fukuda, H., and Yamadori, A. (2002). Encoding-related brain activity during deep processing of verbal materials: a PET study. Neurosci. Res. 44, 429-438.

Grossman, M., Koenig, P., Glosser, G., DeVita, C., Moore, P., Rhee, J., Detre, J., Alsop, D., and Gee, J. (2003). Neural basis for semantic memory difficulty in Alzheimer's disease: an fMRI study. Brain 126, 292-311.

Gurd, J. M., Amunts, K., Weiss, P. H., Zafiris, O., Zilles, K., Marshall, J. C., and Fink, G. R. (2002). Posterior parietal cortex is implicated in continuous switching between verbal fluency tasks: an fMRI study with clinical implications. Brain 125, 1024-1038.

Hickok, G., and Poeppel, D. (2004). Dorsal and ventral streams: a framework for understanding aspects of the functional anatomy of language. Cognition 92, 67-99.

Hickok, G., and Poeppel, D. (2007). The cortical organization of speech processing. Nat. Rev. Neurosci. 8, 393-402.

Howell, D. (2006). Statistical Methods for Psychology, 5th Edn. Australia: Thomson Wadsworth.

Hue, C. W., Kao, C. H., and Lo, M. (2005). Association Norms for 600 Chinese Characters. Taiwanese Psychological Association. Taipei.

Kan, I. P., and Thompson-Schill, S. L. (2004). Effect of name agreement on prefrontal activity during overt and covert picture naming. Cogn. Affect. Behav. Neurosci. 4, 43-57.

Lau, E. F, Phillips, C., and Poeppel, D. (2008). A cortical network for semantics: (de)constructing the N400. Nat. Rev. Neurosci. 9, 920-933.

Leck, K. J., Weekes, B. K., and Chen, M. J. (1995). Visual and phonological pathways to the lexicon: evidence from Chinese readers. Mem. Cognit. 23, 468-476.

Lee, S. H., Chen, S. Y., and Chou, T. L. (in press). Effects of vocabulary sizes on semantic processing to Chinese characters between fifth graders and adults. Formos. J. Ment. Health.

Martin, A. (2007). The representation of object concepts in the brain. Annu. Rev. Psychol. 58, 25-45.

McGregor, K. K., and Appel, A. (2002). On the relation between mental representation and naming in a child with specific language impairment. Clin. Linguist. Phon. 16, 1-20.

McGregor, K. K., Friedman, R. M., Reilly, R. M., and Newman, R. M. (2002). Semantic representation and naming in young children. J. Speech Lang. Hear. Res. 45, 332-346.

Meng, X., Tian, X., Jian, J., and Zhou, X. (2007). Orthographic and phonological processing in Chinese dyslexic children: an ERP study on sentence reading. Brain Res. 1179, 119-130.

Nelson, D. L., McEvoy, C. L., and Schreiber, T. A. (1998). The University of South Florida word association, rhyme, and word fragment norms. http://www. usf.edu/FreeAssociation/.

Paulesu, E., Frith, C. D., and Frackowiak, R. S. J. (1993). The neural correlates of the verbal component of working memory. Nature 362, 342-345.
Peng, D., Guo, D. J., and Zhang, S. L. (1985). Access to the lexical information of Chinese characters during semantic judgment. Acta Psychol. Sin. 3, 227-233.

Penny, W. D., and Holmes, A. (2003). Random effects analysis. In Human Brain Function, 2nd Edn., R. S. J. Frackowiak, K. J. Friston, and C. D. Frith, eds (San Diego, CA, Academic Press), pp. 843-850.

Poldrack, R. A., Wagner, A. D., Prull, M. W., Desmond, J. E., Glover, G. H., and Gabrieli, J. D. (1999). Functional specialization for semantic and phonological processing in the left inferior prefrontal cortex. Neuroimage 10 15-35.

Raposo, A., Moss, H. E., Stamatakis, E. A. and Tyler, L. K. (2006). Repetition suppression and semantic enhancement: an investigation of the neural correlates of priming. Neuropsychologia 44 2284-2295.

Schapiro,M.B.,Schmithorst,V.J.,Wilke,M., Byars, A. W., Strawsburg, R. H., and Holland, S. K. (2004). BOLD fMRI signal increases with age in selected brain regions in children. Neuroreport 15, 2575-2578.

Schmithorst, V. J., Holland, S. K., and Plante, E. (2006). Cognitive modules utilized for narrative comprehension in children: a functional magnetic resonance imaging study. Neuroimage 29, 254-266.

Seger, C. A., Desmond, J. E., Glove, G. H., and Gabrieli, J. (2000). Functional magnetic resonance imaging evidence for right-hemisphere involvement in processing unusual semantic relationships. Neuropsychology 14 361-369.

Sinica Corpus (1998). Academia Sinica Balanced Corpus (version). Taipei. Taiwan: Academia Sinica.

Smith, E. E. (1995). Concepts and categorization. In An Invitation to Cognitive science 2nd Edn., S. M. Kosslyn, D. N Osherson, eds (Cambridge, MA, MIT Press), pp. 1-25.

Song, H., Zhang, H. C., and Shu, H (1995). Developmental changes in functions of orthography and phonology in Chinese reading. Acta Psychol. Sin. 2, 139-144.

Szaflarski, J. P., Schmithorst, V. J., Altaye, M., Byars, A. W., Ret, J. Plante, E., and Holland, S. K. (2006). A longitudinal functional magnetic resonance imaging study of language development in children 5 to 11 years old. Ann. Neurol. 59, 796-807.

Thompson, C. K., Bonakdarpour, B., Fix, S. C., Blumenfeld, H. K., Parrish, T. B., Gitelman, D. R., and Mesulam, M. M. (2007). Neural correlates of verb argument structure processing. J. Cogn. Neurosci. 19 1753-1767.
Thompson-Schill, S. L., D'Esposito, M., Aguirre, G. K., and Farah, M. J. (1997). Role of left inferior prefrontal cortex in retrieval of semantic knowledge: a reevaluation. Proc. Natl. Acad. Sci. U.S.A. 94, 14792-14797.

Thompson-Schill, S. L., D’Esposito, M., and Kan, I. P. (1999). Effects of repetition and competition on activity in left prefrontal cortex during word generation. Neuron 23, 513-522.

Wagner, A. D., Pare-Blagoev, E. J., Clark, J., and Poldrack, R.A.(2001). Recovering meaning: left prefrontal cortex guides controlled semantic retrieval. Neuron 31, 329-338.

Whatmough,C., Chertkow,H., Murtha, S., and Hanratty, K. (2002). Dissociable brain regions process object meaning and object structure during picture naming. Neuropsychologia 40, 174-186.

Wible, C. G., Han, S. D., Spencer, M. H., Kubicki, M., Niznikiewicz, M. H., Jolesz, F. A., McCarley, R. W., and Nestor, P. (2006). Connectivity among semantic associates: an fMRI study of semantic priming. Brain Lang. 97, 294-305.

Wu, J. T., and Liu, I. M. (1987). Exploring the Phonetic and Semantic Features of Chinese Words. Taiwan National Science Council: Technical Report NSC75-0301-H002-024. Taipei.

Zhang, Q., Guo, C. Y., Ding, J. H., and Wang, Z. Y. (2006). Concreteness effects in the processing of Chinese words. Brain Lang. 96, 59-68.

Zhou, X., and Marslen-Wilson, W. (2000). The relative time course of semantic and phonological activation in reading Chinese. J. Exp. Psychol. Learn. Mem. Cogn. 26, 1245-1265.

Conflict of Interest Statement: The authors declare that the research was conducted in the absence of any commercial or financial relationships that could be construed as a potential conflict of interest.

Received: 30 April 2009; paper pending published: 13 June 2009; accepted:06 September 2009; published online: 20 November 2009. Citation: Chou T-L, Chen C-W, Fan L-Y, Chen S-Y and Booth JR (2009) Testing for a cultural influence on reading for meaning in the developing brain: the neural basis of semantic processing in Chinese children. Front. Hum. Neurosci. 3:27. doi 10.3389/neuro.09.027.2009

Copyright (C) 2009 Chou, Chen, Fan, Chen and Booth. This is an open-access article subject to an exclusive license agreement between the authors and the Frontiers Research Foundation, which permits unrestricted use, distribution, and reproduction in any medium, provided the original authors and source are credited. 